\title{
Study to evaluate the immunomodulatory effects of radiofrequency ablation compared to surgical resection for liver cancer
}

\author{
Ketevan Mazmishvili ${ }^{\circledR}$, Kumar Jayant ${ }^{\natural}$, Nona Janikashvili1 ${ }^{1}$, Nino Kikodze ${ }^{1}$, Malkhaz Mizandari², Ia \\ Pantsulaia ${ }^{1}$, Natela Paksashvili 1,2, Mikael H Sodergren ${ }^{3}$, Isabella Reccia ${ }^{3}$, Madhava Pai ${ }^{3}$, Nagy Habib ${ }^{3}$, \\ Tinatin Chikovani ${ }^{1}$ \\ 1. Department of Immunology, Tbilisi State Medical University, 0186 Tbilisi, Georgia \\ 2. Department of Interventional Radiology, Tbilisi State Medical University, High Technology University Clinic, 0144 Tbilisi, Georgia \\ 3. Department of Surgery and Cancer, Hammersmith Hospital, Imperial College London, DuCane Road, W120HS, UK \\ $\triangle$ Corresponding authors: Ketevan Mazmishvili, Department of Immunology, Tbilisi State Medical University, 0186 Tbilisi, Georgia email: \\ keti_mazmishvili@yahoo.com and Kumar Jayant, MD, Department of Surgery and Cancer, Hammersmith Hospital, Imperial College London, DuCane Road, \\ W120HS, UK Tel: +44 (0)20 33138574 Fax: +44 (0)20 33133212 email: jkumar@ic.ac.uk
}

(c) Ivyspring International Publisher. This is an open access article distributed under the terms of the Creative Commons Attribution (CC BY-NC) license (https://creativecommons.org/licenses/by-nc/4.0/). See http://ivyspring.com/terms for full terms and conditions.

Received: 2018.01.22; Accepted: 2018.04.05; Published: 2018.08.06

\begin{abstract}
Introduction: Hepatic cancer is a highly lethal tumour with increasing worldwide incidence. These tumours are characterized by the proliferation of malignant cells, generalised immunosuppression and chronic inflammation marked with an increase in inflammatory markers as a neutrophil-to-lymphocyte ratio (NLR), the platelet-to-lymphocyte ratio (PLR) and overexpression of CD4+CD39+ on T lymphocytes. The studies have outlined immunomodulatory changes in liver cancer patients as the plausible explanation for the better survival. The aim of this pilot study was understand the possible immunomodulatory effect of radiofrequency (RF) energy and liver resection (non-radiofrequency based devices; non-RF device) in relation to NLR, PLR and expression of CD4+CD39+ $T$ lymphocytes and compare the magnitude of these changes.

Material and Methods: In the present study, 17 patients with hepatic cancer were prospectively divided into treatment groups radiofrequency ablation (RFA group) and Liver resection using non-RF devices (LR group). A blood sample was collected from each patient, one month before and after the procedure and compared with the blood samples of age-matched healthy volunteers for group wise comparison. The Mann-Whitney U test, Mc Nemar test and Wilcoxon rank test were used for statistical comparisons as appropriate.

Results: A decrease in NLR was reported after RFA from $4.7 \pm 3.3$ to $3.8 \pm 1.8(P=0.283)$, in contrary to an increase from $3.5 \pm 2.8$ to $4.5 \pm 3.2(P=0.183)$ in $L R$ group. Likewise, a decrease was discerned in PLR following RFA from $140.5 \pm 79.5$ to $137 \pm 69.2$ respectively $(P=0.386)$ and increase in the LR group from $116 \pm 42.2$ to $120.8 \pm 29$ respectively $(P=0.391)$. A significant decrease in $C D 4+C D 39+$ lymphocytes from $55.8 \pm 13.8$ to $24.6 \pm 21.1 \quad(P=0.03)$ was observed in RFA group whilst a significant increase was reported in $L R$ group from $47.6 \pm 8.8$ to $55.7 \pm 33.2(P=0.38)$.

Conclusion: Studies have shown that decrease in the NLR, PLR and expression of CD4+CD39+ on T lymphocytes as the marker of better survival in hepatic cancer patients and our findings have confirmed that these changes can be induced following application of RF energy. Moreover, this could be the explanation of better survival observed in different studies using RFA or other RF-based devices in comparison to non-RF based liver resection techniques. However, further larger studies are needed to confirm these findings.
\end{abstract}

Key words: liver cancer, Hepatocellular carcinoma, Radiofrequency ablation, liver resection, Immunomodulation

\section{Introduction}

Liver cancer is considered as one of the aggressive malignant tumour, a National Cancer Institute and SEER (Surveillance, Epidemiology and
End Result) Program 2016, reported an incidence of 39,230 and a mortality of 27,170 per year [1]. Throughout the world, males are more prone to these 
cancers and disease is 2.1 to 5.7 times more frequent than females [2]. In comparison to the declining death rates observed for various other cancers (including breast, colon, lung), the mortality rates in males for hepatocellular carcinoma arose by $2.8 \%$ per year and for females increased by $3.4 \%$ [1]. The majority of primary liver cancers are hepatocellular carcinomas (HCC), followed by cholangiocarcinoma, gall bladder cancer and so on [3]. It is the third most common cancer of digestive system and the fourth most common cause of cancer-related death following colon and pancreatic cancer [1]. An advancement in the non-surgical and surgical techniques, including radiofrequency ablation (RFA), trans-arterial chemoembolization (TACE), chemotherapy, novel devices for liver resection, liver transplantation and so forth have produced a significant positive impact on the management of patients with liver cancer [4-7]. Nevertheless, prognosis of liver cancer is largely depending on the stage of presentation, and potentially curative treatment options are feasible only at early tumour stages $[8,9]$.

According to Barcelona Clinic Liver Cancer (BCLC) staging system [10,11], liver resection (LR), liver transplantation and percutaneous local ablation including RFA, microwave ablation and so on are recommended as curative therapies for patients with early-stage primary and limited secondary liver tumours $(<3 \mathrm{~cm})$ with a 5 -year survival rate of $50-75 \%$ [12-17]. However, in patients with intermediate HCC or liver metastasis $>3 \mathrm{~cm}$, transarterial chemoembolization (TACE) or transarterial embolization (TAE) in combination with liver resection (LR) may offer a median survival ranging from 16 to 22 month [18-20] (Figure 1). Hence, the use of non-surgical modalities either alone or in combination with surgical resection are often required owing to inadequate functional hepatic reserve, unfavourable anatomical location, stage of liver cancer and multifocal involvement $[21,22]$. Studies demonstrated better long-term survival in the 5 and 10 years' longitudinal studies following RFA for HCC and colorectal liver metastasis [23,24]. In a meta-analysis conducted by $\mathrm{Xu}$ et al., (2014) included 31 studies and total of 16,103 patients concluded that the overall survival and disease free survival was significantly better in RFA group than LR in HCC patients with tumour of $\leq 2 \mathrm{~cm}$ [25]. A recent study by Qiu et al. (2017) has highlighted the benefits of RF based device and reported significantly less morbidity, mortality and better survival over the clamp-crush technique [26]. In addition, Huang et al.

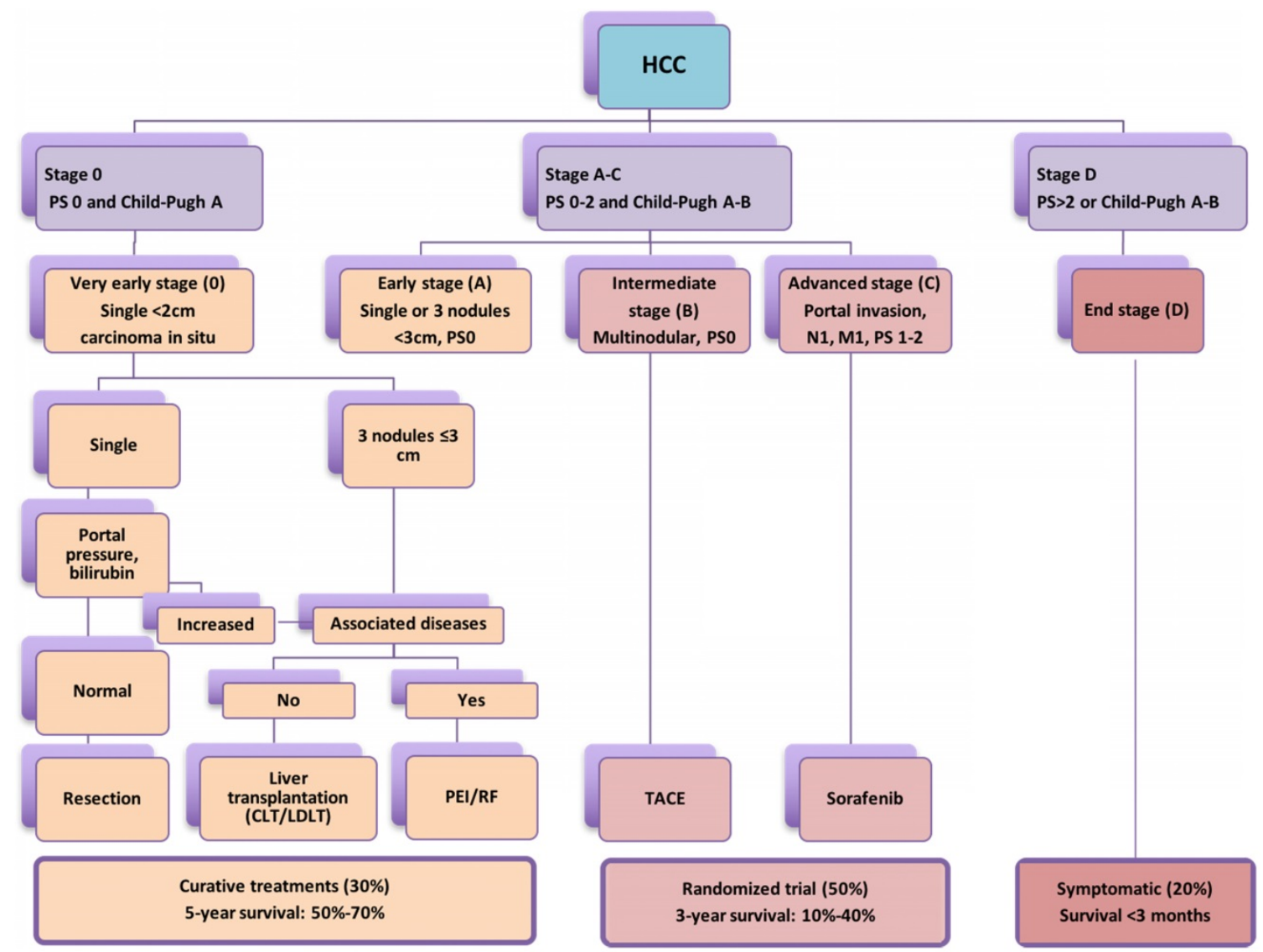

Figure 1. The widely accepted Barcelona Clinic Liver Cancer (BCLC) staging system stating treatment options according to cancer stage, degree of liver dysfunction (Child-Pugh Score) and performance status (PS); CLT: Cadaveric liver transplant; LDLT: Living donor liver transplant. 
(2017) compared RF based liver resection device with cavitron ultrasonic surgical aspirator (CUSA) and outlined significantly better long duration disease-free survival [27]. Further, studies have implicated local and systemic immunomodulatory changes following application of radiofrequency as primary reason behind better survival achieved through RF based approach. The present pilot study was planned to understand the liver tumour biology, observe the immunomodulatory changes following application of RF energy and completed literature analysis for its potential role in advanced liver cancer as an adjunct to check-point inhibitors.

The tumour biology is a systematic concept according to which the behaviour of cancer is not only determined by the genetics of tumour cells but influenced through the microenvironment. Considering the fact that cancer is a state of generalized immunosuppression, considerable evidence evinced, that the tumour site inflammatory reaction can foster its growth and progression. Lately, inflammation-based markers as neutrophil-tolymphocyte ratio (NLR) and platelet-to-lymphocyte ratio (PLR) have been detected in liver cancer patients and received acknowledgment as an important marker of the tumour surveillance system [28-30].

Studies have outlined that increased numbers of neutrophils provide a sustainable microenvironment for liver tumour growth and metastasis through angiogenesis. The relative increase in NLR is considered as the marker of chronic inflammation, and it accounts for tumour characteristics such as vascular invasion and chronic inflammation. The accumulation of neutrophils in the peritumoural stroma enhances tumour invasion through the regulation of a paracrine-mediated hepatocyte growth factor [31-33]. Consequently, proinflammatory cells present in the peritumoral stroma promote the release of IL-17 and other chemokines (CXC) from epithelial cells, which in turn facilitate additional neutrophil and chemokines receptor positive B cells trafficking [34-36]. As mentioned, increased NLR has been found to be associated with poor outcomes in liver cancer patients and considered as an independent predictor for survival following liver resection in HCC [37]. Recent evidence showed that post liver transplant HCC patients have decreased postoperative NLR in comparison to the preoperative NLR with significantly higher serum and peritumoural IL-17 level in the increased NLR group [38]. Concordantly, an inflammatory tumour microenvironment provided by tumour associated macrophages and IL-17 producing cells correlated with HCC recurrence following post liver transplant [39]. In addition, the tumour recurrence following liver transplantation was higher in splenectomised recipients with higher preoperative NLR than those who had not undergone splenectomy. Hence, systemic inflammatory response through upregulation of cytokines, not only promoted hepatic tumour development and growth, but also determined recurrence [40-42]. A recent meta-analysis published by Qi et al. (2017) included 90 papers with 20,475 HCC patients, concluded the importance of NLR in recurrence, disease free survival and overall survival. The changes in NLR during disease course have considerable implication on prognosis too [43].

The platelet-lymphocyte-ratio (PLR) has been explored as a predictor of thrombotic and inflammatory conditions and regarded as an attractive inflammation-related biomarker for liver tumours. Platelets have been shown to induce an epithelial-mesenchymal-like transition and platelet-derived nucleotides and promote tumour cell transendothelial migration and metastasis [44,45]. In a meta-analysis by Zhao et al., (2017) including 10 studies concluded that HCC patients with high PLR have worse overall survival and described them as unfavourable predictors for overall survival [46]. Therefore, both high baseline NLR or PLR have been not only considered as the marker of poor prognosis in patients with liver cancer but also a predictor for cancer invasion, metastasis and survival outcomes [46].

Recognizing that high NLR and PLR are typically associated with chronic inflammatory states, which are linked to immune dysregulation, disordered metabolism, and aberrant cell proliferation in liver cancer. These ratios typically reflect the potential balance between neutrophil and platelet associated pro-tumour systemic inflammation and lymphocyte dependent anti-tumour immune function $[47,48]$. Hence, inflammatory cell and cytokines in tumour microenvironment establish suppressive milieu for cancer growth whilst, dysregulated immune cells help tumour cells in escaping from immune surveillance system.

The immunological perspective of tumour development is a rough house of two wings: an antitumour immunity group i.e CD 8 cells, natural killer (NK) cells, and protumour group including regulatory $\mathrm{T}$ cells (Tregs) and tumour derived repressing substances. The tumour cells escape immunological surveillance by diminished recognition by immune cells, increased resistance by tumour cells, or instigation of an immunosuppressive microenvironment.

An ectonucleotidase (CD 39) which is nucleoside triphosphate diphosphohydrolase- 1 has been discovered on the surface of various cell types 
such as normal leukocytes, Tregs and endothelial cells. Ectonucleotidase CD39 catalyses the hydrolysis of extracellular adenosine triphosphate (ATP) to adenosine. Thus, favours tumour growth as degradation of ATP lowers the immune response $[49,50]$. The upregulation of Tregs has been considered a predictor of poor outcome in HCC patients along with, intratumoural balance of Tregs and cytotoxic T cells determines the prognosis of HCC patients following resection [51,52]. Furthermore, CD39 overexpression in Tregs limits the NK cell activity and promotes the growth of metastatic liver tumours [53,54]. Cai et al. demonstrated increased recurrence and poor overall survival in liver cancer patients with overexpression of CD 39 [55].

In light of the current evidence that decrease in immunomodulatory serum markers including NLR, PLR and expressions of ectonucleotidase CD39 on circulating CD4+ T lymphocytes have potential role in the better survival of liver cancer patients. We decided to conduct a pilot study, to understand the degree of immunomodulatory changes following radiofrequency ablation (RFA) and liver resection (LR) using non-RF device. The pre- and postprocedure, serum NLR, PLR and expressions of ectonucleotidase CD39 on circulating CD4+ $\mathrm{T}$ lymphocytes were analysed to demonstrate the immunomodulatory changes in both treatment groups and in comparison with healthy population.

\section{Material and Methods}

\section{Study Design:}

We conducted a single centre prospective pilot study at Tbilisi State Medical University hospital in accordance with the Declaration of Helsinki from 2014 to 2017. The study was approved by the Ethic Committee of Tbilisi State Medical University (\# $44 / 3$ ). The primary endpoint of study was to assess the immunomodulatory changes in the NLR, PLR and expression of ectonucleotidase CD39 on circulating CD4+ T lymphocytes following RFA or liver resection in the liver cancer patients.

\section{Subjects}

A total of 17 patients with primary (HCC and Cholangiocarcinoma) and secondary (metastatic) liver cancer were enrolled in this prospective study. Out of 17 patients, 7 were referred to the RFA procedure and another 10 underwent surgical liver resection using non RF based devices. All decisions regarding procedures were made following the MDT (Multidisciplinary Team) discussion. Surgical resection was carried out under the general anaesthesia using an upper middle incision. In three patients anatomical segmental hepatectomy while non-anatomical resection was done in rest seven patients. All liver resections were accomplished by using non-RF based liver resection devices. Healthy age-matched volunteers without history of cancer, recent acute or chronic infectious disease, or autoimmune disease, were used as controls for the comparison.

\section{Inclusion and Exclusion criteria}

The inclusion criteria for the RFA patients selection were as follows: a) extensive liver disease or medical co-morbidities associated with tumor vascular invasion and thromboses, b) fewer than three nodules without extrahepatic metastasis, c) largest tumour size of 3-4 cm in diameter, d) visualization of the nodule during the planning of RFA by ultrasonography (US). The exclusion criteria were chemotherapy or TACE, RFA or LR within previous one month.

\section{Technique}

Radiofrequency ablation (RFA): An image-guided RFA was performed by senior interventional radiologist at our centre. The tumour was localized and RF antenna introduced into the target tissue under US guidance. RF processing increases temperature into the target tissue up to $102^{\circ} \mathrm{C}$ leading to the irreversible damage by coagulative necrosis. Abdominal contrast computed tomography (CT) was performed to document completeness of the procedure. Peripheral blood samples were obtained at one month before and after the procedure.

Liver resection (LR): Surgical resection, anatomic segmental hepatectomy and non-anatomical liver resection were carried out under the general anaesthesia using an upper middle incision, using non-RF based liver resection devices.

\section{NLR and PLR counting}

NLR and PLR, known as index systemic inflammatory markers, have proven prognostic role in many types of cancers. Blood samples were collected from each patient in an EDTA anticoagulant-treated tube as per the study protocol. They were further analysed for routine peripheral blood cells, Neutrophil/lymphocyte ratio (NLR) and Platelet/lymphocyte ratio (PLR). The NLR and PLR were defined as the absolute neutrophil count and the absolute platelet count, respectively, divided by the absolute lymphocyte count.

\section{PBMC isolation and Flow cytometry}

Peripheral blood mononuclear cells (PBMCs) were obtained by Ficoll gradient centrifugation. Freshly isolated PBMCs were immediately stained for 
the surface markers using anti-CD4 PE/Cy7 and CD4 subsets were detected by the lineage specific antibodies anti-CD39 PE. Data were acquired on a FacsArray cytometer and analyzed with BD FACSArray system software.

\section{Statistical data analysis}

All data were entered into a Microsoft Excel ${ }^{\mathrm{TM}}$ database and analysed using Graph Pad Prizm software. The Mann-Whitney U test and Mc Nemar test were used to compare data between RFA and surgical resection group. Furthermore, both study groups were compared with healthy control subjects. Wilcoxon's matched pairs signed rank test were used to compare patients before and after therapy. $P$ values less than 0.05 were considered statistically significant.

\section{Results}

\section{Demography}

A total of 17 patients with liver cancer were included in the study. Patients' demographic characteristics of each group has been listed and compared in Table 1 . The mean age of patients in RFA and LR group was $55.1 \pm 11.2$ years and $58.6 \pm 8.1$ years respectively $(P>0.05)$. There were three women $(43 \%)$ and four men $(57 \%)$ in the RFA cohort whilst, five each women and men in the LR group. We didn't observe any significant differences between groups regarding number of tumours primary or secondary, tumour size, tumour stage, $\mathrm{HBsAg}$, Anti-HCV positive.

Table 1: Demographics and Clinical Characteristics of Patients in the Study Groups

\begin{tabular}{llll}
\hline Parameters & $\begin{array}{l}\text { Radiofrequency } \\
\text { Ablation }\end{array}$ & $\begin{array}{l}\text { Liver Resection } \\
\text { (non-RF device) }\end{array}$ & $P$-value \\
\hline Age(years) \pm SD & $55.1 \pm 11.2$ & $58.6 \pm 8.1$ & $>0.05$ \\
No. male/female & $4 / 3$ & $5 / 5$ & $>0.05$ \\
Primary tumours & 1 & 5 & $>0.05$ \\
Secondary tumours & 6 & 5 & $>0.05$ \\
HBsAg-positive, n (\%) & $1(14.2 \%)$ & $1(10.0 \%)$ & $>0.05$ \\
Anti HCV-positive $\mathrm{n}(\%)$ & $0(0 \%)$ & $2(20 \%)$ & \\
Tumour size (mm) \pm SD & $22.4 \pm 5.6 \mathrm{~mm}$ & $47.6 \pm 23 \mathrm{~mm}$ & $<0.05$ \\
Treatment & $7(41.10 \%)$ & $10(58.80 \%)$ & $>0.05$ \\
Localization & $5 \mathrm{R}, 1 \mathrm{~L}, 1 \mathrm{~L} / \mathrm{R}$ & $7 \mathrm{R}, 3 \mathrm{~L}$ & \\
Complication & Hydrodissection & Haemorrhage $(1)$ & $>0.05$ \\
& $(4)$ & & \\
\hline
\end{tabular}

Statistical significance was analyzed by the Mc Nemar Test

Statistical significance was analyzed by the Mann-Whitney U test

Statistical significance was analyzed by the Wilcoxon's matched pairs signed rank test

\section{Neutrophil/lymphocyte ratio (NLR) and platelet/lymphocyte ratio (PLR) in the peripheral blood of patients before and after RFA or Liver resection.}

The post-RFA, NLR assessed in peripheral blood sample were lower but not significantly lower in comparison to pre-RFA levels $(3.8 \pm 1.8$ and $4.7 \pm 3.3$; $P=0.283$ ). However, no such decrease was observed in LR group; pre- and post- procedures levels were $3.5 \pm 2.8$ and $4.5 \pm 3.2$ respectively $(P=0.183)$. Similarly, post-RFA, PLR value $(137 \pm 69.2)$ was lower but not significantly lower than the pre-RFA value (140.5 \pm 79.5$),(P=0.386)$, on the other hand opposite trend was observed in LR group. Here the post-LR group level was higher $(120.8 \pm 29)$ than pre-LR $(116 \pm 42.2)$ but not statistically significant $(P=0.391)$ (Figure 2) (Table 2).
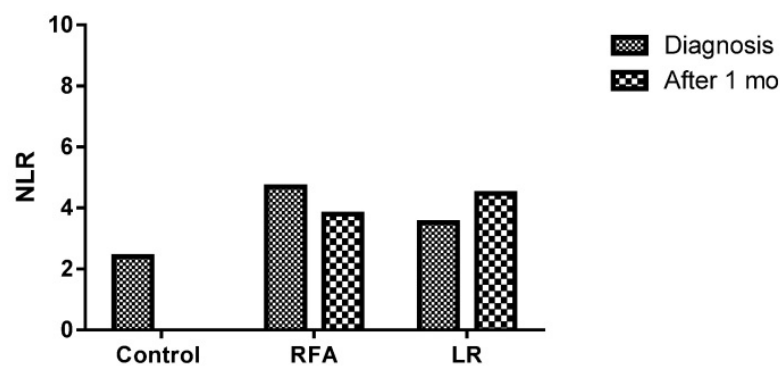
After 1 month

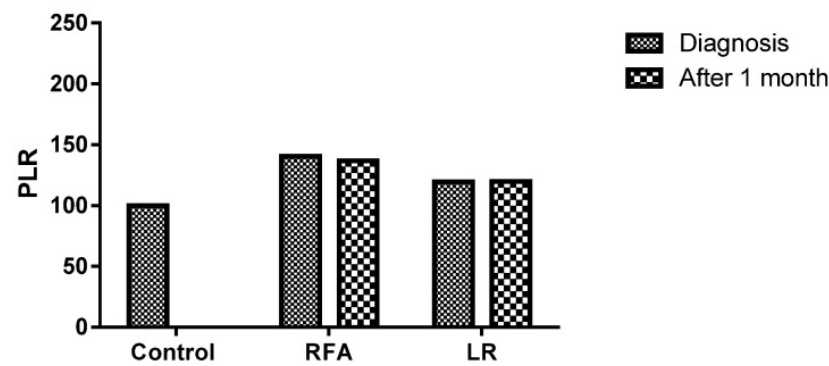

Figure 2. Decreased NLR and PLR in the patients who were treated with RFA (Radiofrequency ablation)

Table 2: Paired comparison of NLR, PLR and CD39+ in the Study Groups

\begin{tabular}{lllllll}
\hline Parameters & NLR & P value & PLR & P value & CD39+ & P value \\
\hline Before RFA & $4.7 \pm 3.3$ & $\mathrm{p}=0.283$ & $140.5 \pm 79.5$ & $\mathrm{p}=0.386$ & $55.8 \pm 13.8$ & $\mathrm{p}=0.03$ \\
After RFA & $3.8 \pm 1.8$ & & $137 \pm 69.2$ & & $24.6 \pm 21.1$ & \\
Before LR & $3.5 \pm 2.8$ & $\mathrm{p}=0.183$ & $116 \pm 42.2$ & $\mathrm{p}=0.391$ & $47.6 \pm 8.8$ & $\mathrm{p}=0.38$ \\
After LR & $4.5 \pm 3.2$ & & $120.8 \pm 29$ & & $55.7 \pm 33.2$ & \\
\hline
\end{tabular}

Statistical significance was analyzed by the Wilcoxon's matched pairs signed rank test

Neutrophil/lymphocyte ratio (NLR)

Platelet/lymphocyte ratio (PLR)

CD39+ (CD39+CD4 T Lymphocytes)

\section{Circulating CD4+CD39+ lymphocytes in the peripheral blood of patients before and after RFA or Liver resection.}

Peripheral blood samples were obtained before and after 1 month of RFA or LR procedure in patients with liver cancer to assess CD4+CD39+ lymphocytes. The significant decrease was observed in RFA group following the procedure while no such difference was seen in LR group.

The CD4+CD39+ lymphocytes levels in RFA group decreased significantly from pre-procedure 
$55.8 \pm 13.8$ to after-procedure 24.6 $\pm 21.1 \quad(P=0.03)$ whilst, in LR group it was $47.6 \pm 8.8$ and $55.7 \pm 33.2$ before and after procedure respectively $(P=0.38)$. The observed value of CD4+CD39+ lymphocytes in healthy controls was $10 \%$.

The short-term effect of the treatment of liver cancer with RFA was decreased expression of CD39 on circulating $\mathrm{CD} 4{ }^{+}$cells however we did not observe the same in the patients treated with surgical resection (Figure 3).
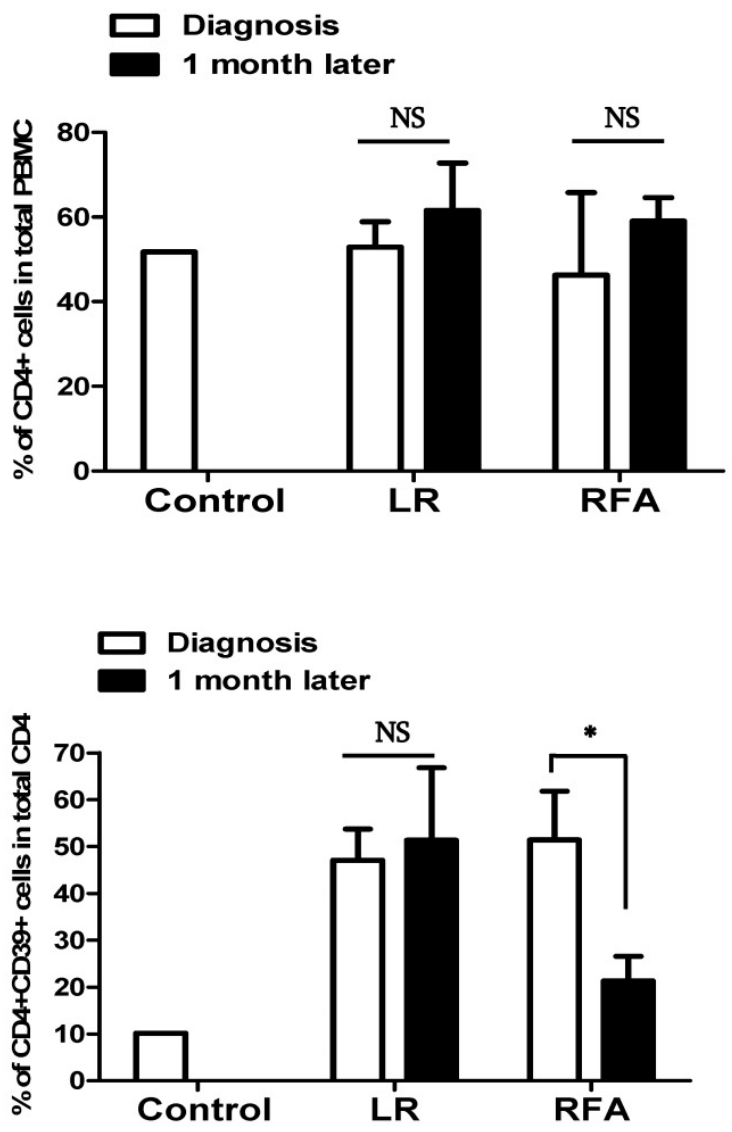

Figure 3. The percentage of $\mathrm{CD} 39+\mathrm{CD} 4+$ cells in total $C D 4+T$ cells was decreased after RFA, but was not modified after LR.

\section{Discussion}

Regardless of the host's underlying condition, chronic inflammation combined with intrinsic cell factors driven by oncogene act as a rheostat in all stages of tumour development; initiation, neoplastic cell growth, proliferation and metastatic spread. A vast majority of experimental work has demonstrated the mechanistic underpinning of host-derived proinflammatory cytokines as inducer of the release of reactive oxygen and nitrogen species, which promote mutational effects and cooperate to instigate epigenetic changes that nurture tumorigenesis [56-60]. This hold true for primary or secondary liver cancers too, which arises and progress in association with chronic inflammation, often linked with immune dysregulation, disordered metabolism and aberrant cell proliferation. Tumour progress and metastasis are not only related to the intrinsic characteristics of cancer cells but also to the cancer microenvironment $[61,62]$.

In a recent meta-analysis done by Qi et al. and Zhao et al. shown that higher NLR and PLR are correlated with adverse survival outcomes in liver cancers patients irrespective of the treatment modalities [43,46]. Recognizing that decrease NLR and PLR are associated with better survival, various studies have reported better disease free survival when radiofrequency based techniques were used in the management of liver cancer patients [27,63-66]. The present study was aimed to assess the immunomodulatory changes in terms of NLR, PLR and percentage of $\mathrm{CD}_{39}{ }^{+} \mathrm{CD} 4{ }^{+}$cells as a possible explanation behind better survival in liver cancer patients receiving RF based treatment.

The present study has showed decrease in post procedural NLR in RFA group however LR group evidenced increase in its level. This was in line to previous studies where higher NLR has been considered as a predictor of cancer recurrence, whilst post treatment lower NLR has been labelled as favourable prognostic factor [43,67-69]. The higher PLR is also considered as an independent prognostic factor and unfavourable predictor of survival in patients with liver cancer [44,70-72]. Here, we observed decrease in post procedural PLR in RFA group in comparison to LR group, which explicate their relationship and usefulness in improving the patient survival and decreasing the risk for tumour recurrence. Studies have elucidated role of anti-inflammatory or anti-platelet medicines in improving the survival of liver cancer patients with higher PLR. The similar attribute may hold plausible explanation for RF either, the post RFA decrease in PLR, not only considered as a better marker for survival but can also bring potential benefit in the management of liver cancer patients with RF based treatment modalities $[73,74]$.

The literature review has demonstrated most intriguing observation of molecule $\mathrm{CD} 39^{+} \mathrm{CD} 4^{+} \mathrm{T}$ cells subsets regarding its ability in inhibition of anti-tumour immune responses and promotion of angiogenesis [75-77]. Cai et al. reported that, CD39 expression in liver cancer can predict postoperative recurrence and survival time in liver cancer patients, thus highlighted the important prognostic value of CD39+ CD4+T cells [55]. In the present research, we studied the changes in CD39 expression of CD4+ T cells and demonstrated significantly lower expression following RFA in contrary LR group. 
Therefore, the NLR, PLR and CD $39{ }^{+} \mathrm{CD} 4{ }^{+} \mathrm{T}$ cells expression must be considered as a panel of potential biomarkers and its level not only reciprocates with systemic inflammation, immune function but also act as an explicit marker to envisage the survival in liver cancer patients. Further, studies demonstrated platelet as a complement of neutrophils in the creation of milieu for cancer angiogenesis and metastasis. The studies have outlined the changes in the tumour microenvironment induced by platelets which help in escaping tumour cells from being phagocytosed by natural killer cells $[75,78,79]$.

However, we didn't assess changes in this cell subtype, but owing to it potential impact on tumour micro environment and immunomodulatory role, it is worth mentioning here. RF produces CD8+ T cells infiltration at the ablation site which helps in phagocytosis of tumour cells debris. The advancement in immunological understanding has led to the development of checkpoint inhibitors drugs tremelimumab which boost CD8+ T cell functioning. The potential effect of these drugs on the immune system is further advantageous in terms of better survival as it acts in synergy with checkpoint inhibitors. In addition, recent trials have shown the synergism in combined use of RF and checkpoint inhibitors could bring more beneficence towards the long-term survival [80-82]. Duffy et al., 2017, conducted a study, where they reported the better survival following combined application of RFA and checkpoint inhibitors. The increased margination of intratumoral CD8 $+\mathrm{T}$ cells at ablation site following RF application and augmentation of their activity by the checkpoint inhibitors demonstrate the synergism of these two therapeutic modalities in the management of advanced aggressive hepatocellular carcinomas [83].

The present study has certain limitations which need to be kept in mind. First, owing to its small sample size and unintended biases of patient selection which might have influenced the analysis. In addition, we didn't assess immunological changes involving other cell types as CD8 and NK cells, which also get modulated following RF application. Nevertheless, we believe that our study has outlined the positive immunomodulatory changes following RFA, which may be suggestive of the better survival observed in liver cancer patients following application of other radiofrequency based techniques too.

\section{Conclusion}

To recapitulate, the decrease in NLR, PLR and significant decrease in CD39+ CD4 T lymphocytes represent a picture of immunomodulatory changes as a possible explanation to the better survival reported by studies using RFA or other RF based techniques in the management of liver cancer patients. However further studies with larger numbers of patients are needed to confirm these preliminary findings.

\section{Acknowledgments}

\section{Ethical approval}

All procedures performed in studies involving human participants were in accordance with the ethical standards of the institutional and/or national research committee and with the 1964 Helsinki declaration and its later amendments or comparable ethical standards.

\section{Funding}

This research was funded by Shota Rustaveli National Science Foundation.

\section{Authors' Contributions}

NJ and TC conceived and designed the study. NJ, KJ, IR and TC screened the abstract and full text, extracted data, assessed studies and drafted the manuscript. NJ and TC performed statistical analyses. $\mathrm{NK}, \mathrm{MM}, \mathrm{KM}, \mathrm{IP}, \mathrm{NP}, \mathrm{MS}, \mathrm{MP}$ and NH revised the manuscript. All authors read the manuscript and approved the final version.

\section{Competing Interests}

Professor Nagy Habib is the inventor of the Habib $^{\mathrm{TM}} 4 \mathrm{X}$ device, which is a radiofrequency based device. None of the other authors have a conflict of interest, including specific financial interests or relationships and affiliations relevant to the subject matter or materials discussed in the manuscript to declare.

\section{References}

[1] Liver cancer incidence and mortality based on SEER data analyzed by National Cancer Institute. 2016.

[2] McGlynn KA, Petrick JL, London WT. Global Epidemiology of Hepatocellular Carcinoma: An Emphasis on Demographic and Regional Variability. Clin. Liver Dis. 2015. p. 223-238.

[3] Ghouri Y, Mian I, Rowe J. Review of hepatocellular carcinoma: Epidemiology, etiology, and carcinogenesis. J. Carcinog. 2017;16:1.

[4] Rahbari NN, Garden OJ, Padbury R, et al. Posthepatectomy liver failure: a definition and grading by the International Study Group of Liver Surgery (ISGLS). Surgery. 2011;149:713-724.

[5] Aragon RJ, Solomon NL. Techniques of hepatic resection. J. Gastrointest. Oncol. 2012;

[6] Kingham TP, Correa-Gallego C, D'Angelica MI, et al. Hepatic parenchymal preservation surgery: Decreasing morbidity and mortality rates in 4,152 resections for malignancy. J. Am. Coll. Surg. 2015.

[7] Asham EH, Kaseb A, Ghobrial RM. Management of Hepatocellular Carcinoma. Surg. Clin. North Am. 2013. p. 1423-1450.

[8] Gish RG. Hepatocellular carcinoma: Current questions and future directions. Gastroenterol. Hepatol. 2015. p. 182-185.

[9] Schlachterman A, Craft WW, Hilgenfeldt E, et al. Current and future treatments for hepatocellular carcinoma. World J. Gastroenterol. 2015;21:8478-8491.

[10] Llovet J, Brú C, Bruix J. Prognosis of Hepatocellular Carcinoma: The BCLC Staging Classification. Semin. Liver Dis. 1999;19:329-338.

[11] D'Avola D, Iñarrairaegui M, Pardo F, et al. Prognosis of hepatocellular carcinoma in relation to treatment across BCLC stages. Ann. Surg. Oncol. 2011;18:1964-1971. 
[12] Kim Y, Lim HK, Rhim H, et al. Ten-year outcomes of percutaneous radiofrequency ablation as first-line therapy of early hepatocellular carcinoma: Analysis of prognostic factors. J. Hepatol. 2013;58:89-97.

[13] Rhim H, Lim HK, Kim YS, et al. Radiofrequency ablation of hepatic tumors: Lessons learned from 3000 procedures. J. Gastroenterol. Hepatol. 2008. p. 1492-1500.

[14] N'Kontchou G, Mahamoudi A, Aout M, et al. Radiofrequency ablation of hepatocellular carcinoma: long-term results and prognostic factors in 235 Western patients with cirrhosis. Hepatology. 2009;50:1475-1483.

[15] Cucchetti A, Piscaglia F, Cescon M, et al. Systematic review of surgical resection vs radiofrequency ablation for hepatocellular carcinoma. World J. Gastroenterol. 2013. p. 4106-4118.

[16] Cucchetti A, Piscaglia F, Cescon M, et al. An explorative data-analysis to support the choice between hepatic resection and radiofrequency ablation in the treatment of hepatocellular carcinoma. Dig. Liver Dis. 2014;46:257-263.

[17] Liu P-H, Hsu C-Y, Hsia C-Y, et al. Surgical Resection Versus Radiofrequency Ablation for Single Hepatocellular Carcinoma $\leq 2 \mathrm{~cm}$ in a Propensity Score Model. Ann. Surg. 2016;263:538-545.

[18] Adhoute X, Penaranda G, Castellani P, et al. Recommendations for the use of chemoembolization in patients with hepatocellular carcinoma: Usefulness of scoring system? World J. Hepatol. 2015;7:521-531.

[19] Barman PM, Su GL. Limitations of the barcelona clinic liver cancer staging system with a focus on transarterial chemoembolization as a key modality for treatment of hepatocellular carcinoma. Clin. Liver Dis. 2016. p. 32-35.

[20] Facciorusso A, Licinio R, Muscatiello N, et al. Transarterial chemoembolization: Evidences from the literature and applications in hepatocellular carcinoma patients. World J. Hepatol. 2015. p. 2009-2019.

[21] Crissien AM, Frenette C. Current management of hepatocellular carcinoma. Gastroenterol. Hepatol. (N. Y). 2014

[22] Sucandy I, Cheek S, Golas BJ, et al. Longterm survival outcomes of patients undergoing treatment with radiofrequency ablation for hepatocellular carcinoma and metastatic colorectal cancer liver tumors. HPB. 2016;18:756-763

[23] Lee DH, Lee JM, Lee JY, et al. Radiofrequency ablation of hepatocellular carcinoma as first-line treatment: long-term results and prognostic factors in 162 patients with cirrhosis. Radiology. 2014;270:900-909.

[24] Kulik LM, Chokechanachaisakul A. Evaluation and Management of Hepatocellular Carcinoma. Clin. Liver Dis. 2015. p. 23-43.

[25] Xu Q, Kobayashi S, Ye X, et al. Comparison of Hepatic Resection and Radiofrequency Ablation for Small Hepatocellular Carcinoma: A Meta-Analysis of 16,103 Patients. Sci. Rep. 2014;4:7252.

[26] Qiu J, Lu W, Yu N, et al. Habib\&lt;sup\&gt;TM\&lt;/sup\&gt; 4X-assisted resection versus clamp-crush resection for hepatocellular carcinoma: a propensity-matching study. Oncotarget. 2017;

[27] Huang K W, Lee P, Kusano T, Reccia I JKHN. Impact of cavitron ultrasonic surgical aspirator (CUSA) and bipolar radiofrequency device (Habib-4X) based hepatectomy for hepatocellular carcinoma on tumour recurrence and disease-free survival. Oncotarget. 2017;55:93644-93654

[28] Li W, Han J, Wu H. Regulatory T-cells promote hepatitis B virus infection and hepatocellular carcinoma progression. Chronic Dis. Transl. Med. 2016;2:67-80.

[29] Hernandez-Gea V, Toffanin S, Friedman SL, et al. Role of the microenvironment in the pathogenesis and treatment of hepatocellular carcinoma. Gastroenterology. 2013. p. 512-527.

[30] Wang X, Hassan W, Jabeen Q, et al. Interdependent and independent multidimensional role of tumor microenvironment on hepatocellular carcinoma. Cytokine. 2017;

[31] Kwon H-C, Kim SH, Oh SY, et al. Clinical significance of preoperative neutrophil-lymphocyte versus platelet-lymphocyte ratio in patients with operable colorectal cancer. Biomarkers. 2012;17:216-222.

[32] Wu G, Yao Y, Bai C, et al. Combination of platelet to lymphocyte ratio and neutrophil to lymphocyte ratio is a useful prognostic factor in advanced non-small cell lung cancer patients. Thorac. Cancer. 2015:6.275-287.

[33] Zhang H, Xia H, Zhang L, et al. Clinical significance of preoperative neutrophil-lymphocyte vs platelet-lymphocyte ratio in primary operable patients with non-small cell lung cancer. Am. J. Surg. 2015;210:526-535.

[34] Kuang D-M, Peng C, Zhao Q, et al. Tumor-Activated Monocytes Promote Expansion of IL-17-Producing CD8+ T Cells in Hepatocellular Carcinoma Patients. J. Immunol. 2010;185:1544-1549.

[35] Zhao Q, Xiao X, Wu Y, et al. Interleukin-17-educated monocytes suppress cytotoxic T-cell function through B7-H1 in hepatocellular carcinoma patients. Eur. J. Immunol. 2011;41:2314-2322.

[36] Kuang DM, Peng C, Zhao Q, et al. Activated monocytes in peritumoral stroma of hepatocellular carcinoma promote expansion of memory $\mathrm{T}$ helper 17 cells. Hepatology. 2010;51:154-164

[37] Yamamura $\mathrm{K}$, Sugimoto $\mathrm{H}$, Kanda $\mathrm{M}$, et al. Comparison of inflammation-based prognostic scores as predictors of tumor recurrence in patients with hepatocellular carcinoma after curative resection. J. Hepatobiliary. Pancreat. Sci. 2014;21:682-688.

[38] Motomura T, Shirabe K, Mano Y, et al. Neutrophil-lymphocyte ratio reflects hepatocellular carcinoma recurrence after liver transplantation via inflammatory microenvironment. J. Hepatol. 2013;58:58-64.

[39] Chen T-M, Lin C-C, Huang P-T, et al. Neutrophil-to-lymphocyte ratio associated with mortality in early hepatocellular carcinoma patients after radiofrequency ablation. J. Gastroenterol. Hepatol. 2012;27:553-561.
[40] Galdiero MR, Bonavita E, Barajon I, et al. Tumor associated macrophages and neutrophils in cancer. Immunobiology. 2013;218:1402-1410.

[41] Coussens LM, Werb Z. Inflammation and cancer. Nature. 2002. p. 860-867.

[42] Jaiswal M, LaRusso NF, Burgart LJ, et al. Inflammatory cytokines induce DNA damage and inhibit DNA repair in cholangiocarcinoma cells by a nitric oxide-dependent mechanism. Cancer Res. 2000;60:184-190.

[43] Qi X, Li J, Deng H, et al. Neutrophil-to-lymphocyte ratio for the prognostic assessment of hepatocellular carcinoma: A systematic review and meta-analysis of observational studies. Oncotarget. 2016;7:45283-45301.

[44] Templeton AJ, McNamara MG, Šeruga B, et al. Prognostic role of neutrophil-to-lymphocyte ratio in solid tumors: A systematic review and meta-analysis. J. Natl. Cancer Inst. 2014.

[45] Labelle M, Begum S, Hynes RO. Direct Signaling between Platelets and Cancer Cells Induces an Epithelial-Mesenchymal-Like Transition and Promotes Metastasis. Cancer Cell. 2011;20:576-590.

[46] Zhao Y, Si G, Zhu F, et al. Prognostic role of platelet to lymphocyte ratio in hepatocellular carcinoma: a systematic review and meta-analysis. Oncotarget. 2017:8:22854-22862.

[47] Jablonska J, Leschner S, Westphal K, et al. Neutrophils responsive to endogenous IFN-beta regulate tumor angiogenesis and growth in a mouse tumor model. J. Clin. Invest. 2010;120:1151-1164.

[48] Crespo J, Sun H, Welling TH, et al. T cell anergy, exhaustion, senescence, and stemness in the tumor microenvironment. Curr. Opin. Immunol. 2013. p. 214-221.

[49] Ostrand-Rosenberg S, Sinha P, Beury DW, et al. Cross-talk between myeloid-derived suppressor cells (MDSC), macrophages, and dendritic cells enhances tumor-induced immune suppression. Semin. Cancer Biol. 2012. p. 275-281.

[50] Croci DO, Zacarías Fluck MF, Rico MJ, et al. Dynamic cross-talk between tumor and immune cells in orchestrating the immunosuppressive network at the tumor microenvironment. Cancer Immunol. Immunother. 2007. p. 1687-1700.

[51] Deaglio S, Dwyer KM, Gao W, et al. Adenosine generation catalyzed by CD39 and CD73 expressed on regulatory $\mathrm{T}$ cells mediates immune suppression. J. Exp. Med. 2007;204:1257-1265.

[52] Borsellino G, Kleinewietfeld M, Di Mitri D, et al. Expression of ectonucleotidase CD39 by Foxp3+ Treg cells: Hydrolysis of extracellular ATP and immune suppression. Blood. 2007;110:1225-1232.

[53] Gao Q, Qiu S-J, Fan J, et al. Intratumoral balance of regulatory and cytotoxic T cells is associated with prognosis of hepatocellular carcinoma after resection. J. Clin. Oncol. 2007:25:2586-2593.

[54] Sun X, Wu Y, Gao W, et al. CD39/ENTPD1 expression by CD4+Foxp3+ regulatory $\mathrm{T}$ cells promotes hepatic metastatic tumor growth in mice. Gastroenterology. 2010;139:1030-1040.

[55] Cai X-Y, Ni X-C, Yi Y, et al. Overexpression of CD39 in hepatocellular carcinoma is an independent indicator of poor outcome after radical resection. Medicine (Baltimore). 2016;95:e4989.

[56] Grivennikov SI, Greten FR, Karin M. Immunity, Inflammation, and Cancer. Cell. 2010. p. 883-899.

[57] Karin M, Lawrence T, Nizet V. Innate immunity gone awry: Linking microbial infections to chronic inflammation and cancer. Cell. 2006. p. 823-835.

[58] Vesely MD, Kershaw MH, Schreiber RD, et al. Natural Innate and Adaptive Immunity to Cancer. Annu. Rev. Immunol. 2011;29:235-271.

[59] Sun B, Karin M. Obesity, inflammation, and liver cancer. J. Hepatol. 2012;56:704-713.

[60] Zhong Z, Sanchez-Lopez E, Karin M. Autophagy, Inflammation, and Immunity: A Troika Governing Cancer and Its Treatment. Cell. 2016. p. 288-298.

[61] Weber A, Boege $\mathrm{Y}$, Reisinger $\mathrm{F}$, et al. Chronic liver inflammation and hepatocellular carcinoma: Persistence matters. Swiss Med. Wkly. 2011.

[62] Sun X, Han L, Seth P, et al. Disordered purinergic signaling and abnormal cellular metabolism are associated with development of liver cancer in Cd39/ENTPD1 null mice. Hepatology. 2013;57:205-216.

[63] I. S, S. C, B.J. G, et al. Longterm survival outcomes of patients undergoing treatment with radiofrequency ablation for hepatocellular carcinoma and metastatic colorectal cancer liver tumors. HPB. 2016;18:756-763.

[64] Morimoto M, Numata K, Kondou M, et al. Midterm outcomes in patients with intermediate-sized hepatocellular carcinoma: A randomized controlled trial for determining the efficacy of radiofrequency ablation combined with transcatheter arterial chemoembolization. Cancer. 2010;116:5452-5460.

[65] Feng K, Yan J, Li X, et al. A randomized controlled trial of radiofrequency ablation and surgical resection in the treatment of small hepatocellular carcinoma. J. Hepatol. 2012;57:794-802.

[66] Prieto J, Melero I, Sangro B. Immunological landscape and immunotherapy of hepatocellular carcinoma. Nat. Rev. Gastroenterol. Hepatol. 2015.

[67] Dan J, Zhang Y, Peng Z, et al. Postoperative Neutrophil-to-Lymphocyte Ratio Change Predicts Survival of Patients with Small Hepatocellular Carcinoma Undergoing Radiofrequency Ablation. PLoS One. 2013.

[68] Oh BS, Jang JW, Kwon JH, et al. Prognostic value of C-reactive protein and neutrophil-to-lymphocyte ratio in patients with hepatocellular carcinoma. BMC Cancer. 2013;13.

[69] Gomez D, Farid S, Malik HZ, et al. Preoperative neutrophil-to-lymphocyte ratio as a prognostic predictor after curative resection for hepatocellular carcinoma. World J. Surg. 2008;32:1757-1762 
[70] Xia W, Ke Q, Wang Y, et al. Predictive value of pre-transplant platelet to lymphocyte ratio for hepatocellular carcinoma recurrence after liver transplantation. World J. Surg. Oncol. 2015;13.

[71] Lee S, Loecher M, Iyer R. Immunomodulation in hepatocellular cancer. J. Gastrointest. Oncol. 2018.

[72] Peng W, Li C, Zhu WJ, et al. Prognostic value of the platelet to lymphocyte ratio change in liver cancer. J. Surg. Res. 2015;194:464-470.

[73] Hossain MA, Kim DH, Jang JY, et al. Aspirin induces apoptosis in vitro and inhibits tumor growth of human hepatocellular carcinoma cells in a nude mouse xenograft model. Int. J. Oncol. 2012;40:1298-1304.

[74] Protasiewicz M, Szymkiewicz P, Kuliczkowski W, et al. Modern antiplatelet therapy - Opportunities and risks. Adv. Clin. Exp. Med. 2013. p. 875-885.

[75] Whiteside TL. The tumor microenvironment and its role in promoting tumor growth. Oncogene. 2008. p. 5904-5912.

[76] Chaudhary B, Elkord E. Regulatory T Cells in the Tumor Microenvironment and Cancer Progression: Role and Therapeutic Targeting. Vaccines. 2016;4:28.

[77] Gu J, Ni X, Pan X, et al. Human CD39hi regulatory T cells present stronger stability and function under inflammatory conditions. Cell. Mol. Immunol. 2017;14:521-528

[78] Yan MJ, Jurasz P. The role of platelets in the tumor microenvironment: From solid tumors to leukemia. Biochim. Biophys. Acta - Mol. Cell Res. 2016. p. 392-400.

[79] Fuentes E, Palomo I, Rojas A. Cross-talk between platelet and tumor microenvironment: Role of multiligand/RAGE axis in platelet activation. Blood Rev. 2016. p. 213-221.

[80] Tumeh PC, Harview CL, Yearley JH, et al. PD-1 blockade induces responses by inhibiting adaptive immune resistance. Nature. 2014;515:568-571.

[81] Xu W, Jiang $\mathrm{H}$, Gao J, et al. The upregulation of immune checkpoint ligand PD-L1 in tumour microenvironment. Scand. J. Immunol. 2014. p. 71-72.

[82] Hiroishi K, Eguchi J, Baba T, et al. Strong CD8(+) T-cell responses against tumor-associated antigens prolong the recurrence-free interval after tumor treatment in patients with hepatocellular carcinoma. J. Gastroenterol. 2010;45:451-458.

[83] Duffy AG, Ulahannan S V., Makorova-Rusher O, et al. Tremelimumab in combination with ablation in patients with advanced hepatocellular carcinoma. J. Hepatol. 2017 